VIII ${ }^{\text {èmes }}$ Journées Nationales Génie Civil - Génie Côtier, Compiègne, 7-9 septembre 2004

\title{
Modélisation 3D d'un pieu chargé latéralement dans un massif sableux à proximité d'un talus
}

\author{
Fidèle Massamba $^{(\mathrm{a})}$, Salim Mezazigh $^{(\mathrm{b})}$, Daniel Levacher $^{(\mathrm{c})}$ \\ ${ }^{(a)}$ Doctorant, ${ }^{(b)}$ Maître de Conférences, ${ }^{(c)}$ Professeur: \\ Université de Caen \\ M2C-GRGC-UMR 6143 CNRS : 24, rue des Tilleuls, 14000 CAEN \\ fidèle.massamba@geos.unicaen.fr
}

\begin{abstract}
Résumé
La réaction d'un sol horizontal sur un pieu est souvent modélisée par des lois d'interaction qui représentent plus ou moins correctement la réalité. Cependant, l'effet d'un talus à proximité du pieu est difficile à quantifier et les recommandations du fascicule 62 (1993), sont à ce sujet assez discutables. On présente ici une analyse numérique 3D et les possibilités du logiciel DIANA-Delft pour le calcul de la réponse d'un pieu soumis à des sollicitations latérales à proximité d'un talus. Une étude (Mezazigh,1995) sur modèle réduit centrifugé est décrite et les données expérimentales recueillies constituent une base de données qui serviront à une confrontation numérique-expérimentale. L'analyse numérique linéaire est menée avec un choix de maillage effectué à l'aide du logiciel DIANA-Delft et l'utilisation des courbes de réaction P-y issues de la base de données. D'importants écarts sont constatés entre les valeurs expérimentales et celles issues de la modélisation numérique. Il est alors fait état de modifications à opérer pour obtenir une meilleure validation.
\end{abstract}

\begin{abstract}
The horizontal reaction soil on a pile is often modelled by interaction laws, that closely represents the soil conditions on site. However, the slope effect near the pile is difficult to quantify and the recommendations of French Foundation Codes (Fascicule 62, 1993) are rather ambiguous. Centrifuge modelling test results and the Finite Element Method (FEM) results are compared in the present study. FEM was carried out using DIANA and the centrifuge test results used here have been reported by Mezazigh (1995). Comparative results show an important difference that may be linked to the FEM development which is based on linear model.
\end{abstract}

\section{Introduction}

Les fondations profondes sur pieux verticaux sont devenues des solutions courantes pour reprendre les efforts horizontaux. Elles peuvent être sollicitées latéralement par des charges statiques comme c'est le cas des efforts d'amarrage sur des quais, des poussées de terre sur des murs de soutènement ou des culées de pont. La présence d'une pente à proximité d'un pieu rend le comportement du système pieu-sol plus difficile à appréhender du fait que la surface du sol n'est plus horizontale. De plus, le problème est tridimensionnel et la nonlinéarité du comportement du sol rend le problème complexe à étudier. Cependant les études sur le comportement d'un pieu isolé chargé latéralement sont nombreuses et restent toujours d'actualité tant sur le plan théorique qu'expérimental. Elles utilisent très souvent des méthodes dites au module de réaction dans lesquelles le sol est idéalisé par des séries de ressorts horizontaux non-linéaires. Ces méthodes sont basées sur les courbes de réaction P-y qui lient, dans chaque couche, la réaction du sol $\mathrm{P}$ au déplacement horizontal y du pieu. Ainsi, 
une étude pour déterminer l'influence de la proximité d'une pente sur un pieu a été menée suivant une approche expérimentale à partir d'une modélisation en centrifugeuse effectuée au LCPC de Nantes et d'une approche numérique en s'appuyant sur un modèle tridimensionnel d'un pieu isolé chargé latéralement à partir d'une méthode en éléments finis.

\section{$\underline{\text { 2.Etude expérimentale }}$}

Des essais ont été réalisés pour étudier le comportement de pieux fichés dans un massif de sable à différentes densités relatives et soumis à des charges latérales. Les pieux prototypes utilisés sont considérés comme longs et flexibles. Sur les pieux modèles, des profils de moments fléchissants ont été déterminés expérimentalement par l'intermédiaire de jauges de déformation collées tout au long du pieu. Ainsi, une série de 59 essais a été menée (Mezazigh, 1995) sur des modèles réduits de pieux centrifugés pour deux pentes de talus $(2 / 1$ et $3 / 2)$, correspondant respectivement à des angles de $26.6^{\circ}$ et $33.7^{\circ}$. Les essais ont été réalisés dans un sable de Fontainebleau sec. Le pieu modèle est constitué d'un tube en aluminium de $380 \mathrm{~mm}$ de long et de $18 \mathrm{~mm}$ de diamètre (B), instrumenté de 20 paires de jauges espacées de $15 \mathrm{~mm}$, installées à l'extérieur du pieu. La fiche (D) est de $300 \mathrm{~mm}$. Les essais ont été réalisés à $40 \mathrm{~g}$. Les déplacements en tête du pieu sont mesurés à l'aide de deux capteurs. Un autre capteur mesure l'effort appliqué en tête. Les paramètres considérés dans l'étude sont : l'angle de talus, la distance relative par rapport au talus (t/B) et la densité du massif. Etant donné le nombre de paramètres à étudier et le nombre d'essais à effectuer, les auteurs affirment que l'approche expérimentale par centrifugation est l'une des méthodes qui permet le mieux de simuler correctement un état de contrainte et de déformation entre le pieu modèle et le pieu prototype. Pour le sable constituant le sol d'implantation des pieux, on peut rappeler quelques caractéristiques physiques. Elles sont résumées dans le tableau 1 ci-dessous.

Tableau 1: Poids volumiques et indice de vides caractéristiques du sable de Fontainebleau

\begin{tabular}{|c|c|c|c|c|}
\hline$\gamma_{\mathbf{s}}\left[\mathrm{kNm}^{-3}\right]$ & $\gamma_{\mathbf{d} \min }\left[\mathrm{kNm}^{-3}\right]$ & $\gamma_{\mathbf{d} \max }\left[\mathrm{kNm}^{-3}\right]$ & $\mathbf{e}_{\min }$ & $\mathbf{e}_{\max }$ \\
\hline 26.44 & 13.64 & 16.83 & 0.616 & 0.940 \\
\hline
\end{tabular}

Auparavant, les essais de Bouafia (1990) sur l'effet de la proximité d'un talus effectués sur des modèles réduits en centrifugeuse ont montré que les moments fléchissants dans le pieu ne dépendent ni de la teneur en eau du massif, ni du mode d'installation du pieu dans le sable ni de la rugosité à l'interface pieu-sol. Mais les déplacements en tête du pieu sont fortement influencés par la densité et la rugosité du pieu. La pente utilisée lors de ces essais était de 2/1 avec une hauteur de talus allant jusqu'à $3 \mathrm{~m}$. Selon Bouafia, l'influence se fait sentir jusqu'à une distance de 6 à 7B pour des pieux rigides.

\section{Etudes théoriques}

La complexité de l'interaction pieu-sol à proximité d'une pente et la diversité des paramètres physiques expliquent la rareté des analyses théoriques. Mezazigh et Levacher (2002) se sont intéressés à l'étude d'un pieu chargé latéralement à proximité d'un talus dans un massif sableux. Les déplacements du pieu sont obtenus par une double intégration de la courbe des moments fléchissants et les constantes d'intégration sont déduites des mesures en tête du déplacement du pieu. Par contre, la double dérivation des moments fléchissants permet de déterminer les courbes de pression $\mathrm{P}(\mathrm{z})$. Un logiciel de lissage utilisant des fonctions splines ou des polynômes a été développé afin d'aboutir à des résultats corrects et validés. Les courbes de réaction P-y (figure 1) sont ensuite déduites point par point et pour chaque 
profondeur à partir des courbes précédentes $(\mathrm{P}(\mathrm{z}), \mathrm{y}(\mathrm{z}))$. Ces courbes présentent une forme parabolique et peuvent être définies par l'expression analytique suivante:

$\mathrm{P} \cdot \mathrm{B}=\alpha\left(\mathrm{I}_{\mathrm{D}}\right) \cdot \frac{\mathrm{Z}}{\mathrm{B}} \cdot\left(\frac{\mathrm{y}}{\mathrm{B}}\right)^{0,7}$

avec $\alpha$, coefficient déterminé en fonction de l'indice de densité $I_{D}$ du sol.

Pour un pieu flexible ou souple ayant une rigidité relative $K_{r}$ de $10^{-5}$, il existe une distance limite du pieu à la crête du talus, de l'ordre de $10 \mathrm{~B}$, au delà de laquelle les déplacements du pieu ne sont plus influencés par la présence du talus.

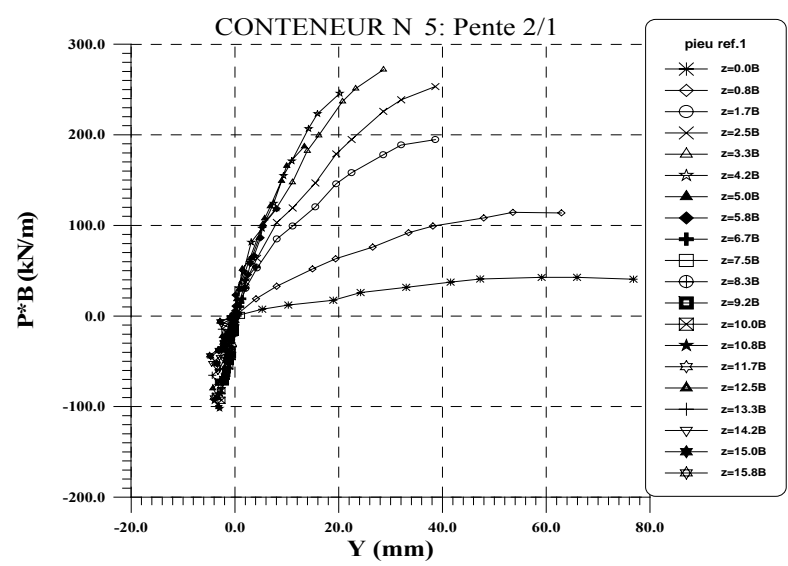

Figure 1 : Courbe de réaction P-y expérimentale

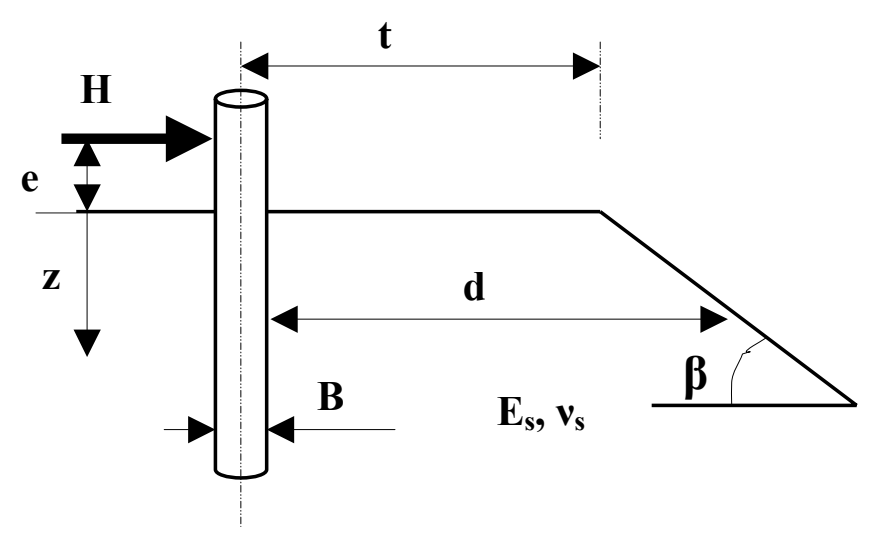

\section{Liste des symboles}

$\mathrm{d}$ : distance du pieu au talus

e : excentricité de la charge

$\mathrm{t}$ : distance à la crête du talus

$\mathrm{z}$ : profondeur dans le sol

$\mathrm{B}$ : diamètre du pieu

$\mathrm{D}$ : fiche du pieu

$\mathrm{H}$ : charge latérale appliquée

$\mathrm{I}:$ inertie du pieu

$\mathrm{E}_{\mathrm{s}}$ : module de réaction du sol

$\beta$ : angle du talus

$v_{\mathrm{s}}$ : coefficient de Poisson

Figure 2 : Schéma d'un pieu implanté à proximité d'un talus

\subsection{Fascicule 62}

Le règlement français (fascicule 62, 1993) recommande une méthode simple de correction de la courbe de réaction latérale P-y basée sur les essais pressiométriques afin de tenir compte de la présence du talus. La méthode stipule que, pour les sections du pieu distantes d'au moins 5B par rapport au talus, la courbe P-y est celle du sol horizontal. Par contre pour les distances inférieures, le fascicule 62 recommande de minorer uniquement les valeurs de la réaction horizontale ultime par un facteur géométrique $\mathrm{z}_{1} / \mathrm{z}_{2}, \mathrm{z}_{2}$ est la profondeur de la section du pieu distante de $5 \mathrm{~B}$ du talus. L'origine des profondeurs est le point fictif qui correspond à l'intersection de la ligne du talus avec l'axe du pieu comme le montre la figure 3. 

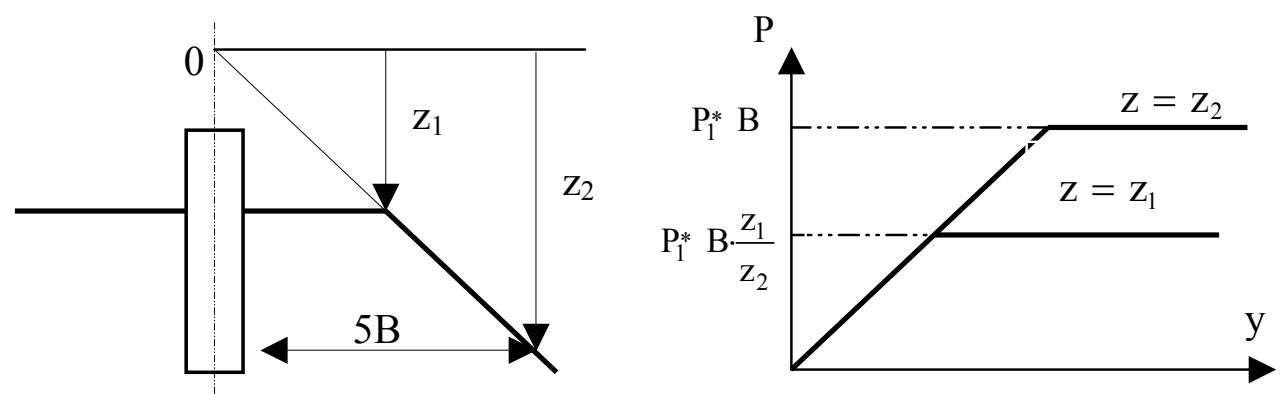

Figure 3: Courbe P-y modifiée, fascicule 62 (1993)

\subsection{Influence de la distance d'un pieu à la crête de talus}

A partir d'études expérimentales, Mezazigh et Levacher (2002), ont déterminé l'influence de la présence d'un talus sur les déplacements en tête pour une pente de $2 / 1$. Les observations faites sont les suivantes : le rapport entre le déplacement d'un pieu situé à une distance t/B et le déplacement en tête du pieu de référence soumis à la même charge ne dépend pas de la charge appliquée, il est d'environ 1.6 pour un talus de $2 / 1$ et d'environ 2.4 pour un talus $3 / 2$. Pour le talus de $2 / 1$, l'effet de pente ne se fait plus sentir pour des distances supérieures à $6 \mathrm{~B}$ ou 7B. Pour un talus de 3/2, les distances limites d'influence sont d'environ 10 à 12B.

\subsection{Calcul des valeurs du coefficient de réduction}

On détermine les valeurs du coefficient de réduction $\mathrm{r}$ des courbes de réaction $\mathrm{P}$-y à partir des données expérimentales de Mezazigh (1995). Puis, elles sont comparées avec les propositions du fascicule 62 (1993) et confrontées à la nouvelle proposition faite par Mezazigh et Levacher (2002). En sachant que le coefficient de réduction $r$ varie en fonction de plusieurs paramètres, les courbes de réaction sont tracées en fonction de $d$ (figure 2) qui prend en compte la profondeur $\mathrm{z}$, la distance du pieu au talus $\mathrm{t}$ et la tangente $\beta$ du talus :

$\mathrm{d}=\mathrm{t}+\frac{\mathrm{z}}{\tan \beta}$

avec une distance limite $d_{c}=5 B$, le coefficient de réduction est établi comme suit :

$\mathrm{r}=\frac{\mathrm{t} \tan \beta+\mathrm{z}}{\mathrm{d}_{\mathrm{c}} \tan \beta}$ pour $\quad 0 \leq \mathrm{z} \leq \mathrm{d}_{\mathrm{c}} \tan \beta-\mathrm{t} \tan \beta$

$\mathrm{r}=1 \quad$ pour $\quad \mathrm{z} \geq \mathrm{d}_{\mathrm{c}} \tan \beta-\mathrm{t} \tan \beta$

Par ailleurs, l'analyse des résultats de dépouillement des essais réalisés par Mezazigh et Levacher (2002) pour deux pentes considérées (2/1 et 3/2) ont conduit à la proposition suivante du coefficient de réduction:

- pour la pente 2/1, avec une distance limite $d_{c}=8 B$

$\mathrm{r}=0.5(1-\tan \beta)\left(1+\frac{3 \mathrm{~d}}{\mathrm{~d}_{\mathrm{c}}} \frac{\tan \beta}{1-\tan \beta}\right)$ pour $0 \leq \mathrm{d} \leq \mathrm{d}_{\mathrm{c}}$ 
- pour la pente $3 / 2$, avec une distance limite $d_{c}=12 B$

$\mathrm{r}=0.85 \times 0.5(1-\tan \beta)\left(1+\frac{3 \mathrm{~d}}{\mathrm{~d}_{\mathrm{c}}} \frac{\tan \beta}{1-\tan \beta}\right)$ pour $0 \leq \mathrm{d} \leq \mathrm{d}_{\mathrm{c}}$

\section{Modélisation numérique}

\subsection{Utilisation du logiciel DIANA-Delft et choix du maillage}

L'objectif est de réaliser un modèle de maillage par éléments finis qui tien compte à la fois de la géométrie du pieu et du massif, et de la capacité de calcul disponible. Dans ce but, le logiciel DIANA-Delft (2000) a été utilisé pour la modélisation 3D. Il peut effectuer des calculs par la méthode des éléments finis de pieux chargés latéralement. Afin de réduire la taille du système pieu-sol étudié, le pieu et le massif du sol sont divisés en deux parties symétriques. Ils sont respectivement modélisés par des éléments isoparamétriques, des pentaèdres à 6 nœuds et des hexaèdres à 8 nœuds. Le logiciel DIANA-Delft (2000) impose quelques critères au niveau des dimensions de chaque élément du maillage :

- pour chaque face, les triangles doivent ressembler le plus possible à des triangles équilatéraux, les quadrilatères à des carrés et les hexaèdres à des cubes,

- la taille des éléments doit rester telle que le rapport de la plus petite dimension de l'élément sur sa plus grande dimension ne soit pas inférieure à 0.12 ,

- pour les pentaèdres, les angles doivent rester entre $30^{\circ}$ et $150^{\circ}$,

- pour les hexaèdres, les angles doivent rester entre $30^{\circ}$ et $140^{\circ}$.

Selon les différents critères imposés par le logiciel DIANA-Delft (2000), des modifications se sont donc avérées importantes pour la modélisation et l'obtention du maillage. L'épaisseur du pieu étant très faible, ceci permet de respecter le rapport de dimensions des éléments de 0.12 imposé par le logiciel.

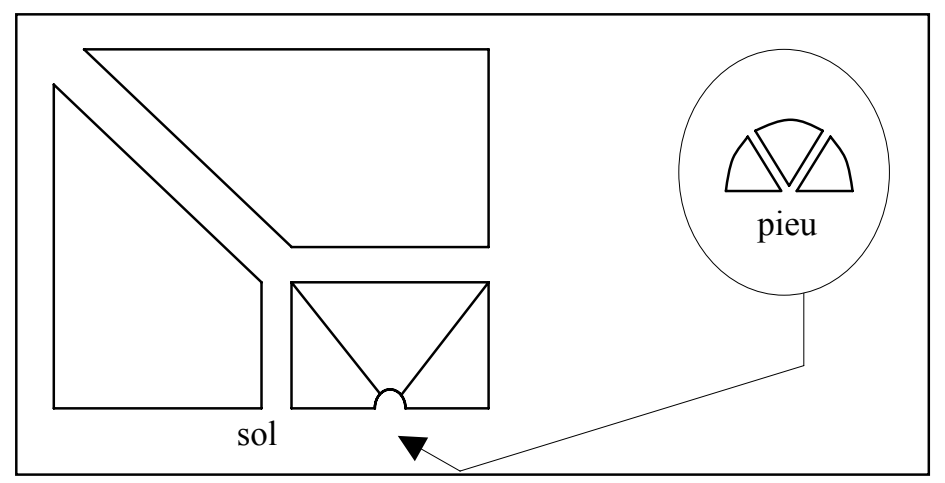

Figure 4: Découpage du massif du sol et du pieu

Afin de contourner cette difficulté, le pieu cylindrique creux a été remplacé par un pieu cylindrique plein, mais tout en conservant la même rigidité $E_{p} I_{p}\left(473.610^{6} \mathrm{Nm}^{2}\right)$ et la même inertie $\mathrm{I}_{\mathrm{p}}\left(22.5510^{-4} \mathrm{~m}^{4}\right)$. Nous avons donc défini le nouveau diamètre du pieu par la relation:

$\mathrm{I}_{\mathrm{p}}=\pi \mathrm{B}^{4} / 64$ soit $\mathrm{B}=\sqrt[4]{64 \mathrm{I}_{\mathrm{p}} / \pi}=463 \mathrm{~mm}$ 
La fiche du pieu est subdivisée en 10 sous-éléments selon les recommandations de Rajashree et Sitharam (2001) et de Kimura et al., (1991). L'excentricité e du pieu, voir figure 2 , est partagée en 4 sous-éléments selon le rapport de forme de 0.12 qu'impose le logiciel DIANA-Delft (2000). La demie circonférence du pieu, voir figure 4, est découpée en 3 parties en l'associant au découpage du massif du sol. L'intervalle limite pour les angles oblige à découper le massif de sol en un demi-carré autour du pieu pour ensuite le compléter autour en conservant des angles de l'ordre de $45^{\circ}$, comme le montre la figure 4 . Le talus $2 / 1$, n'a pas été modélisé du fait que la pente correspond à un angle de $26.6^{\circ}$, donc inférieur à $30^{\circ}$, que le logiciel DIANA-Delft (2000) ne peut pas prendre en compte. Par contre ce critère a permis de s'intéresser et de modéliser la pente $3 / 2$ dont l'angle, $33.7^{\circ}$ est supérieur à $30^{\circ}$. Afin d'obtenir un maillage homogène entre les différents découpages, le talus a été découpé en 2 parties comme illustré sur la figure 5 .

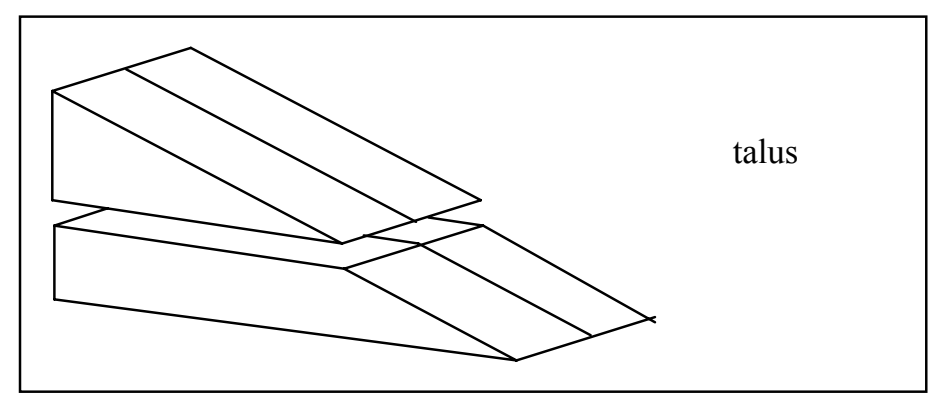

Figure 5: Découpage du talus 3/2 en 2 parties

Le modèle est limité à une distance de $5 \mathrm{~m}$ autour du pieu ce qui correspond à une distance supérieure à 10B. A cette distance, le sol n'est plus influencé par le chargement du pieu, il n'y donc aucun déplacement du sol. Les trois paramètres pris en compte pour la simulation numérique dans le logiciel DIANA-Delft (2000) sont récapitulés dans le tableau 2.

Tableau 2: Caractéristiques du pieu et du sol

\begin{tabular}{|c|c|c|c|}
\cline { 2 - 4 } \multicolumn{1}{c|}{} & Module d'élasticité & Masse volumique & Coefficient de Poisson \\
\hline sol & $\mathrm{E}_{\mathrm{s}}=25 \mathrm{MPa}$ & $\rho_{\mathrm{s}}=1539 \mathrm{~kg} / \mathrm{m}^{3}$ & $v_{\mathrm{s}}=0.33$ \\
\hline pieu & $\mathrm{E}_{\mathrm{p}}=2.110^{5} \mathrm{MPa}$ & $\rho_{\mathrm{p}}=7800 \mathrm{~kg} / \mathrm{m}^{3}$ & $\nu_{\mathrm{p}}=0.285$ \\
\hline
\end{tabular}

\subsection{Calculs et analyse}

Après avoir établi la géométrie du système pieu-sol et maillé ses constituants à l'aide du logiciel DIANA-Delft (2000), les calculs sont effectués par le logiciel et s'ensuit une analyse des résultats. 


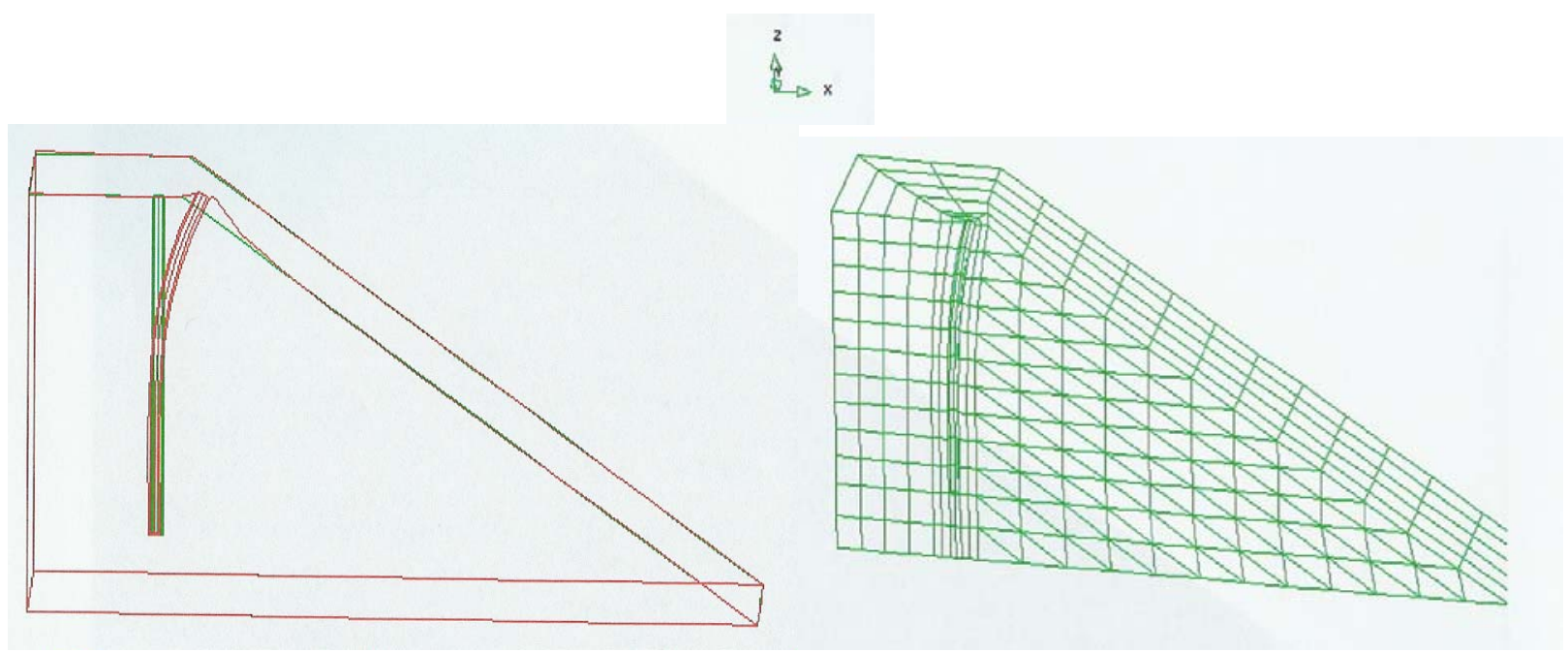

Figure 6: Maillage complet du système pieu-sol-talus

Tout d'abord, il faut indiquer au logiciel le type des éléments de maillage qu'il doit utiliser et le type de calcul qu'il doit faire. Pour se rapprocher au mieux de la réalité, il faut effectuer un calcul en considérant un comportement non-linéaire du système pieu-sol. De plus, le sol est décomposé en plusieurs sous-couches de caractéristiques différentes. La version du logiciel DIANA-Delft (2000) disponible n'est pas capable de prendre en compte le comportement non-linéaire, nous nous sommes limités à des calculs linéaires. Le système pieu-sol est relativement simple et se résume à 908 nœuds et 1024 éléments pour son maillage.

\subsection{Comparaison des courbes de déplacement et des moments fléchissants}

Les profils de moments fléchissants pour les six incréments de chargement appliqués en tête ainsi que les profils de déplacements du pieu sont tracés sur la figure 7. On s'aperçoit que l'allure des courbes est identique à celles obtenues par l'expérimentation en centrifugeuse. Toutes les courbes passent par un point maximum situé à la même profondeur.

Dans cette étude, le sol a été modélisé en 10 sous-couches d'épaisseur constante et le pieu par 14 tronçons de mêmes caractéristiques géométriques et mécaniques. Les incréments de charge latérale $(141 \mathrm{kN}, 130 \mathrm{kN}, 213 \mathrm{kN}, 216 \mathrm{kN}, 283 \mathrm{kN}$ et $366 \mathrm{kN})$ ont été appliquées au milieu de la partie émergente du pieu. Les résultats concernant les déplacements et les moments fléchissants du pieu sont présentés à la figure 7. 

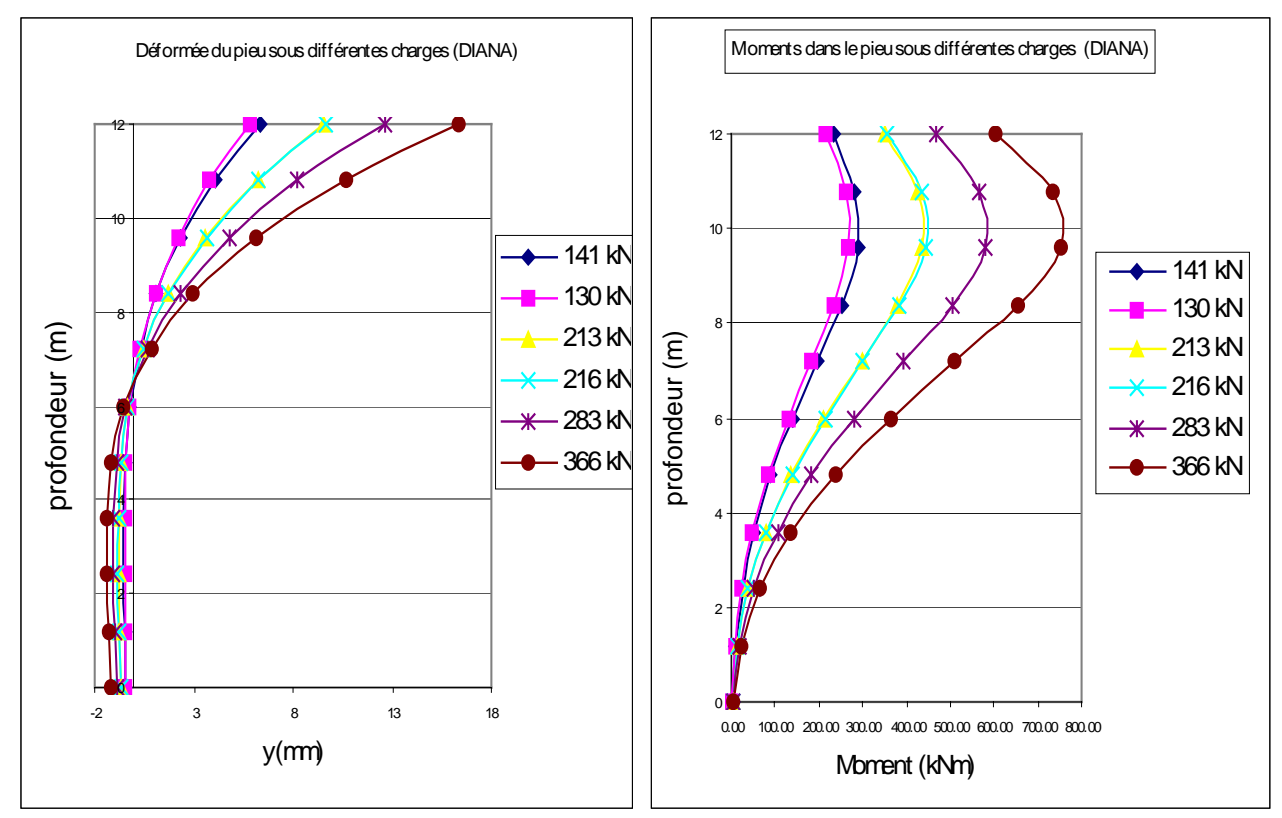

Figure 7 : Profils de déplacements et de moments fléchissants du pieu

\subsection{Ecarts entre les valeurs expérimentales et numériques}

Le tableau 3 montre que d'importants écarts ont été observés entre les valeurs expérimentales et numériques à l'aide du logiciel DIANA-Delft (2000). Ces écarts sont de l'ordre de $50 \%$ sur les déplacements maxima du pieu à la surface du sol et de l'ordre de $30 \%$ pour les moments fléchissants maxima. Ces différences d'écart s'expliquent surtout par les calculs en comportement élastique linéaire qui minimisent les déplacements, car le sol a tendance à retenir le pieu qui se déforme. Aussi le maillage choisi reste assez grossier.

Tableau 3: Ecarts observés entre valeurs numériques et issues de l'expérience

\begin{tabular}{|c|c|c|c|c|c|c|c|}
\cline { 2 - 8 } \multicolumn{1}{c|}{} & \multicolumn{3}{c|}{ DIANA-Delft } & \multicolumn{2}{c|}{ EXPERIENCE } & \multicolumn{2}{c|}{ ECARTS } \\
\hline $\begin{array}{c}\mathbf{H} \\
{[\mathrm{kN}]}\end{array}$ & $\begin{array}{c}\text { Y surface } \\
{[\mathrm{mm}]}\end{array}$ & $\begin{array}{c}\boldsymbol{\sigma}_{\max } \\
{\left[\mathrm{MNm}^{-1}\right]}\end{array}$ & $\begin{array}{c}\mathbf{M}_{\max } \\
{[\mathrm{kN} . \mathrm{m}]}\end{array}$ & $\begin{array}{c}\text { Y surface } \\
{[\mathrm{mm}]}\end{array}$ & $\begin{array}{c}\mathbf{M}_{\max } \\
{[\mathrm{kN} . \mathrm{m}]}\end{array}$ & $\begin{array}{c}\text { Y surface } \\
{[\mathrm{mm}]}\end{array}$ & $\begin{array}{c}\mathbf{M}_{\max } \\
{[\mathrm{kN} . m]}\end{array}$ \\
\hline 141.344 & 6.32 & 29.7 & 289.4 & 11.85 & 390.1 & $46.7 \%$ & $25.8 \%$ \\
\hline 130.832 & 5.85 & 27.5 & 268.0 & 12.15 & 395.9 & $51.9 \%$ & $32.3 \%$ \\
\hline 213.536 & 9.54 & 44.9 & 437.5 & 19.43 & 602.7 & $50.9 \%$ & $27.4 \%$ \\
\hline 216.544 & 9.68 & 45.5 & 443.4 & 20.35 & 624.4 & $52.4 \%$ & $29.0 \%$ \\
\hline 283.456 & 12.70 & 59.6 & 580.7 & 29.29 & 832.4 & $56.6 \%$ & $30.2 \%$ \\
\hline 366.912 & 16.40 & 77.1 & 751.3 & 40.69 & 1105.0 & $59.7 \%$ & $32.0 \%$ \\
\hline
\end{tabular}




\section{Conclusions}

On a réalisé une modélisation numérique du système pieu-sol-talus à l'aide d'un logiciel de calcul par éléments finis. Ces calculs ont été effectués en comportement linéaire avec un maillage grossier et en l'absence d'éléments d'interfaces. Un des objectifs était de confronter les courbes de réaction P-y numériques et celles obtenues lors d'une modélisation expérimentale en centrifugeuse par Mézazigh (1995). Un maillage simplifié a été réalisé et a permis d'aboutir à des résultats très éloignés de la réalité en terme d'amplitude, mais semblables à ceux obtenus par l'expérimentation au niveau du comportement. Un autre objectif était d'étudier les possibilités du logiciel de calcul DIANA-Delft (2000). Cette étude n'a pas permis d'aboutir aux objectifs souhaités vu les difficultés rencontrées dans la modélisation numérique et les limites du logiciel. L'étude sera poursuivie par une modélisation 3D par éléments finis d'un système pieu-sol sous chargement statique et cyclique avec le logiciel ANSYS. On étudiera le comportement d'un pieu dans un sol multicouche avec le module de réaction du sol variable dans chaque sous-couche, différents angles de talus et d'autres paramètres pouvant influencer le système sol-pieu lors d'une sollicitation latérale statique et cyclique.

\section{Références}

1.Mezazigh S. (1995). Etude expérimentale de pieux chargés latéralement : proximité d'un talus et effet de groupe. Thèse de doctorat. Université de Nantes, $217 \mathrm{p}$.

2.Bouafia A. (1990). Modélisation des pieux chargés latéralement en centrifugeuse. Thèse de doctorat en génie-civil, Université de Nantes, $267 \mathrm{p}$.

3.Mezazigh S., Levacher D. (2002). Design of laterally single pile in sand near a slope - a proposal for subgrade reaction reduction factors. $9^{\text {ème }}$ Conférence Internationale sur les Pieux et les Travaux de Fondations Profondes, DFI 2002, Nice Acropolis 3-5 juin 2002.

4.Fascicule $\mathrm{N}^{\circ} 62$ (1993). Règles techniques de conception et de calcul des fondations des ouvrages de géni-civil. Géotechnique, Edition Eyrolles, 188p.

5.DIANA-Delft (2000). User's manual, Pre-and postprocessing. TNO Building and Construction Research, Division of Engineering Mechanics and information Technology. June 2000-Third Edition.

6.Rajashree S. S. et Sitharam T. G. (2001). Non-linear finite-element modelling of batter piles under lateral load. Journal of Geotechnical and Geoenvironmental Engineering. Vol. 127, $\mathrm{N}^{\circ}$ 7, July 2001. ASCE, pp 604-612.

7.Kimura M., Yashima A., Shibata T. (1991). Three-dimensional finite element analysis of laterally loaded piles. Computer Methods and Advances in Geomechanics. Proceedings of $7^{\text {th }}$ International Conference 1991. pp 145-150. 